\title{
Gain-Dependent Polarization Properties of Vertical-Cavity Lasers
}

\author{
Kent D. Choquette, Richard P. Schneider, Jr., Kevin L. Lear, Member, IEEE, and Ronald E. Leibenguth
}

\begin{abstract}
We show that the partitioning of power into the two orthogonal eigen polarizations of infra-red gain-guided verticalcavity lasers depends upon the relative spectral overlap of the nondegenerate polarization cavity resonances with the laser gain spectrum. Furthermore, at the condition where the polarization resonances and the peak laser gain are aligned, abrupt switching of power between the eigen polarizations is observed as the gain sweeps through the polarization resonances. The gain-dependence of the polarization requires spectral splitting between the eigen polarizations, which is found to be strongly influenced by local strain. The polarization of the fundamental and higher-order spatial modes can be selected and maintained for all InGaAs vertical-cavity lasers in a wafer simply by employing a $20 \mathrm{~nm}$ or greater blue-shift offset of the peak laser gain relative to the cavity resonances.
\end{abstract}

\section{INTRODUCTION}

D UE to their topology, vertical-cavity surface emitting lasers (VCSEL's) are particularly suitable for applications requiring two-dimensional laser arrays. Maintaining a constant and consistent polarization state for all laser diodes in an array will be critical for the deployment of VCSEL's in polarization-sensitive applications. However, this can be particularly challenging in a VCSEL since each electromagnetic field distribution possesses two linear orthogonal transverse electric eigen polarizations which can share the laser output power. [1], [2]. Moreover, higher-order transverse modes tend to be orthogonally polarized from the dominant fundamental polarization state [3]. These two effects can conspire to produce polarization partitioning noise [2] and/or an unspecified polarization state in the VCSEL output.

Several methods to produce a single dominant polarization state in a VCSEL have been considered [4]-[9]. All of the schemes rely in some manner on breaking the symmetry in the plane of the quantum wells [4], such as by introducing anisotropic gain[5], [6] or loss [7], [8] to the laser, or by using an anisotropic transverse cavity geometry [9]. With differing degrees of success, these methods promote a single polarization state for the threshold transverse spatial mode, but at the expense of increased fabrication complexity. Even if polarization control is maintained near threshold, higher-order transverse modes still tend to remain orthogonally polarized.

Manuscript received November 1, 1994; revised January 14, 1995. The work performed at Sandia National Laboratories is supported by the U. S. Department of Energy under contract No. DE-AC04-94AL85000.

K. D. Choquette, R. P. Schneider, Jr., and K. L. Lear are with Sandia National Laboratories, P.O. Box 5800, MS 060, Albuquerque, NM 87185 USA.

R. E. Leibenguth is with AT\&T Bell Laboratories, STC, Breinigsville, PA 18031 USA.

IEEE Log Number 9411066.
Fundamentally, what governs the competition between the VCSEL eigen polarizations, especially in the absence of an intentional anisotropy, is unclear.

Polarization modulation between the eigen polarizations of a given VCSEL transverse mode has also been reported [10]-[12]. The polarization modulation frequency should not be limited by the carrier recombination lifetime and should occur with little or no chirp. Inducing the dominant polarization to switch to the orthogonal state has been accomplished by current modulation about a polarization switching transition [10], or through polarized optical feedback to the VCSEL [11], [12]. For both of these polarization modulation schemes, it is important to understand the characteristics of the VCSEL eigen polarizations.

We report a systematic study of the polarization characteristics of infra-red gain-guided circular VCSEL's. The power partitioning into the eigen polarizations is shown to be related to the relative spectral overlap of the nondegenerate polarization cavity resonances with the laser gain spectrum. Furthermore, we show that polarization switching transitions arise when the polarization resonances and the peak laser gain are spectrally aligned. The influence of the cavity resonance/gain alignment is studied using intentionally nonuniform VCSEL wafers and temperature tuning. The polarization gaindependence is found to require a spectral splitting between the polarization cavity resonances to produce a sufficient difference in gain to allow one or the other eigen polarization to dominate. The gain-dependence of the VCSEL polarization provides a simple means to implement polarization control in VCSEL arrays.

\section{EXPERIMENT}

In this study we examine InGaAs quantum-well VCSEL's grown by metalorganic vapor phase epitaxy [13] (MOVPE) and GaAs quantum-well VCSEL's grown by molecular beam epitaxy (MBE) nominally designed for top emission at 950 and $850 \mathrm{~nm}$, respectively. The VCSEL wafers consist of epitaxial distributed Bragg reflector (DBR) mirrors surrounding a one wavelength thick active region. The structural differences between the MBE and MOVPE wafers are the compositions of the low and high index layers in the DBR (AlAs-- $\mathrm{Al}_{0.16} \mathrm{Ga}_{0.84} \mathrm{As}$ for the $850 \mathrm{~nm}$ MBE wafers versus $\mathrm{Al}_{0.96} \mathrm{Ga}_{0.04} \mathrm{As}-\mathrm{GaAs}$ for the $980 \mathrm{~nm}$ MOVPE wafers), the grading between these layers ("staircase" for the MBE wafers versus a parabolic profile for the MOVPE wafers [13]) and the composition, and strain of the quantum wells. The MBE wafers are grown on (100) substrates and are rotated dur- 
ing growth. The MOVPE wafers are grown on misoriented (100) substrates tilted $6^{\circ}$ toward $\langle 111\rangle \mathrm{A}$ to enhance dopant incorporation [14]. The MOVPE wafers are intentionally not rotated during growth to produce a $7 \%$ variation of layer thickness and thus cavity resonance wavelength, but only a $1 \%$ change in the peak gain wavelength, along the direction of gas flow. The substrates are placed in the MOVPE reactor such that the gas flow is parallel with a $\langle 110\rangle$ crystal axis. The wafer nonuniformity enables examination of VCSEL's possessing a wide variation of spectral overlap between the cavity resonance wavelength and laser gain as a function of the device position on the wafer.

Planar gain-guided VCSEL's, $15 \mu \mathrm{m}$ in diameter, are fabricated using proton implantation to funnel the current into the active region. The top electrical contacts have $10 \mu \mathrm{m}$ diameter apertures. The lasers are tested on a probe station and care is taken to insure that minimum pressure is applied by the probe tip on the top contact during the measurements. Polarizationresolved light intensity versus current (L-I) measurements are made on numerous VCSEL samples fabricated from several different wafers. The L-I measurements are done over a broad range of heat sink temperatures, from $100-350 \mathrm{~K}$. The spectral splittings between the eigen polarizations of the 850 nm VCSEL's are determined using a scanning Fabry-Perot interferometer with a resolution of approximately $0.8 \mathrm{GHz}$.

\section{RESULTS}

Above lasing threshold, we typically observe the linearly polarized VCSEL output to have an intensity maximum and minimum in orthogonal directions, corresponding to the eigen polarizations. The eigen polarizations of the GaAs VCSEL's have a random orientation in the plane of the quantum wells, which can vary between neighboring lasers and vary with repeated measurements and/or operating temperature for a given laser [2]. In contrast, the InGaAs VCSEL's in this study exhibit eigen polarizations that are always parallel with the $\langle 110\rangle$ crystal axes (denoted as 0 and $90^{\circ}$ ) over a wide range of temperatures. It has been shown that substrate misorientation away from (100) will remove the VCSEL gain degeneracy in the $\langle 110\rangle$ crystal directions [4]. However, we have found that GaAs VCSEL wafers grown on misoriented substrates do not have a fixed polarization orientation to the crystal axes [15]. Furthermore, for the nonuniform InGaAs VCSEL wafers examined here, the variation in layer thicknesses and/or strain in the quantum well may also contribute to the polarization orientation. In any event, while the substrate misorientation, wafer nonuniformity, and/or quantum-well strain may create sufficient anisotropy to align the eigen polarizations with the crystallographic axes, we show in the following that it does not influence the partitioning of the light into the two eigen polarizations.

Fig. 1 shows the polarized and unpolarized room temperature L-I curves of InGaAs VCSEL's from various locations on a wafer along its centerline parallel to the gas flow direction. In Fig. 1 both the cavity resonance/gain alignment and the dominant threshold polarization state vary with wafer location. For this same wafer we plot in Fig. 2(a) the threshold current, $I_{\mathrm{th}}$, threshold wavelength, and dominant threshold eigen polarization versus the device location. The origin in Fig. 2 corresponds to the "downstream" edge of the wafer with relatively thinner layers and thus the shortest cavity resonance. In this region of the wafer, the laser gain spectrum is red-shifted relative to the cavity resonance, and the $0^{\circ}$ eigen polarization consistently dominates for all devices, as in Fig. 1(a). Conversely, at the opposite "upstream" end of the wafer, corresponding to blue-shifted gain, the $90^{\circ}$ eigen polarization consistently dominates as shown in Fig. 1(c). In between these regions, near the vicinity of the wafer with lasers exhibiting minimum $I_{\text {th }}$, the $90^{\circ}$ state initially dominates until an abrupt switching transition where upon the opposite polarization prevails as displayed in Fig. 1(b). Note the first polarization switching transition occurs before the onset of a higher-order spatial mode which occurs at the arrow in Fig. 1(b).

The polarization partitioning behavior manifest in Fig. 2(a) has been observed in numerous other InGaAs and GaAs VCSEL wafers. For example, Fig. 2(b) shows the VCSEL polarization properties of another InGaAs wafer with reduced top mirror reflectivity compared to the wafer in Fig. 2(a). As a result of the lower output coupler reflectivity, the operating wavelength range of VCSEL's across the wafer in Fig. 2(b) is less than that of the wafer in Fig. 2(a). However, notice that identical polarization characteristics are observed: Each eigen polarization is dominant in regions of the wafer on either side of the minimum $I_{t h}$, and a region of polarization switching lies between.

Fig. 3 depicts the temperature dependence of $I_{t h}$ and the dominant threshold polarization for a GaAs VCSEL [2]. In Fig. 3, the spectral mismatch between the cavity resonance and gain is varied by their noncommensurate thermal shifts [16] rather than by layer thickness nonuniformity as in Fig. 2. The particular GaAs VCSEL in Fig. 3 at room temperature has red-shifted gain offset relative to the cavity resonance; thus as the device is cooled the laser gain can be adjusted to produce a blue-shifted gain offset. We again observe that the eigen polarizations are each dominant at temperatures on opposite sides of the minimum $I_{\mathrm{th}}$, separated by a temperature region where the VCSEL exhibits polarization switching. Identical behavior to that shown in Fig. 3 is also seen in InGaAs VCSEL's. The inset in Fig. 3 shows the spectral splitting at room temperature between the eigen polarizations of the Gaussian mode as measured by a Fabry-Perot interferometer. The two peaks in the inset, which correspond to the two eigen polarizations, are separated by $9.9 \mathrm{GHz}(0.23 \AA)$ which remains constant with current, voltage, or temperature variations. The spectral splitting between the eigen polarizations is influenced by local strain, as described below.

In Fig. 4(a), we show $I_{\text {th }}$ and the threshold wavelength for devices from another GaAs VCSEL wafer. The origin in Fig. 4(a) corresponds to the wafer center, where the laser gain is blue-shifted relative to the cavity resonance; near the wafer edge the cavity resonance and gain are aligned producing the lowest $I_{\mathrm{th}}$. Fig. 4(b) shows the dominant threshold polarization while the VCSEL's are tested under "equilibrium" conditions, i.e., with minimum pressure applied by the probe 


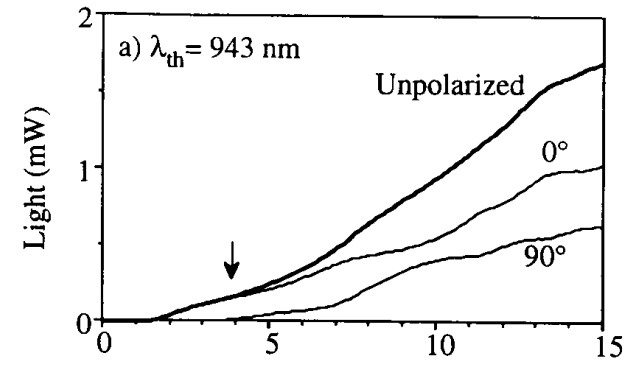

(a)

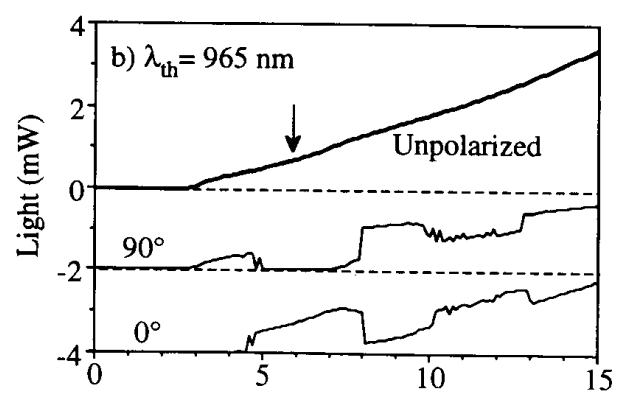

(b)

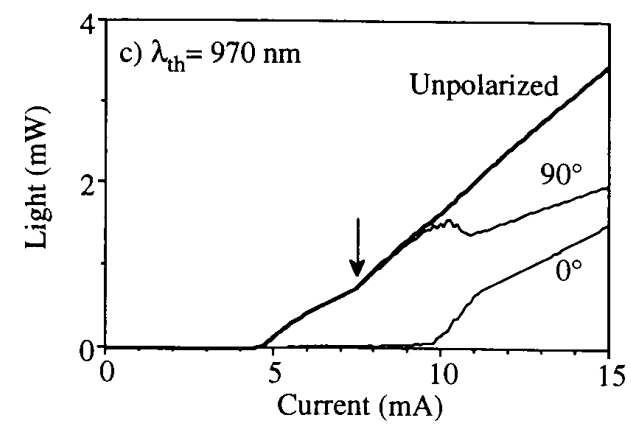

(c)

Fig. 1. Unpolarized (thick curve) and polarized (thin curves) laser emission versus current at room temperature for three InGaAs VCSEL's at different locations in wafer XD1112: (a) $\lambda_{\mathrm{th}}=943 \mathrm{~nm}$, (b) $\lambda_{\mathrm{th}}=965 \mathrm{~nm}$ (curves offset for clarity), and (c) $\lambda_{t h}=970 \mathrm{~nm}$. The eigen polarizations are aligned along the $\langle 110\rangle$ crystal directions denoted as 0 and $90^{\circ}$, and the arrows denote the onset of a higher-order transverse optical mode.

tip. Note the dominant polarization does not exhibit the same correlation with the cavity resonance/gain alignment as seen in Fig. 2. In fact, for most devices, both polarization states are simultaneously observed at and slightly above threshold, with only small differences in their relative partitioning. Also shown in Fig. 4(b) are the lasing lineshapes measured by the Fabry-Perot interferometer slightly above threshold from VCSEL's at three wafer locations; the splitting between the eigen polarizations is found to be $<1.3 \mathrm{GHz}(<0.03 \AA)$. In Fig. 4(c), we show the dominant polarization and the observed emission lineshapes under strained probing conditions, i.e. pressure is applied to the top contact with the probe tip. Notice in Fig. 4(c) that the polarization gain-dependence is observed using the strained probing conditions, along with a significant increase in the spectral splitting between the eigen polarizations. From examination of several GaAs VCSEL samples from the same

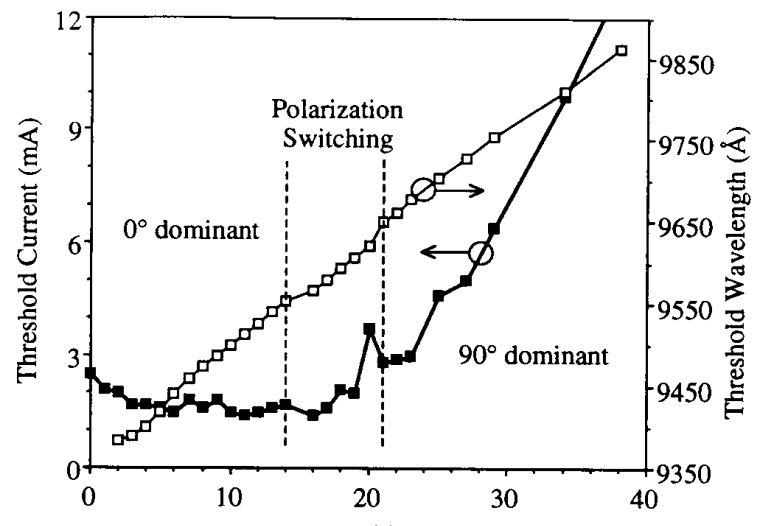

(a)

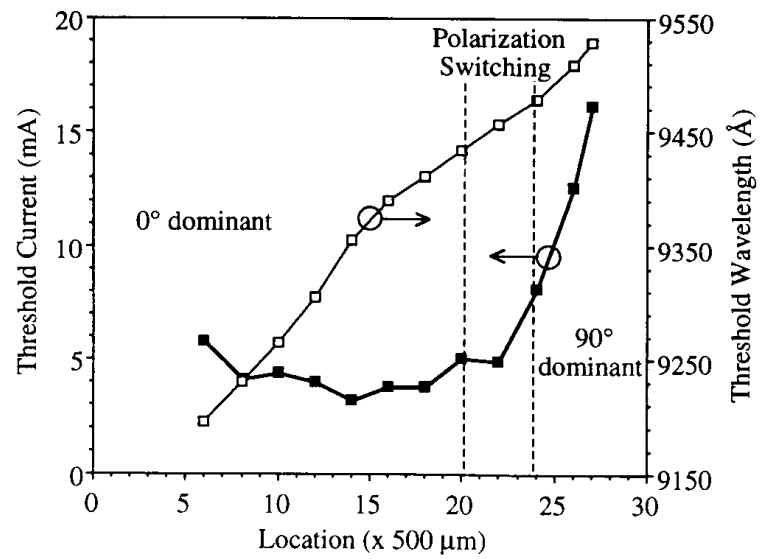

(b)

Fig. 2. Injection current, wavelength, and dominant polarization at threshold versus wafer location for: (a) InGaAs VCSEL wafer XD1112; and (b) InGaAs VCSEL wafer XD1115, which has reduced output mirror reflectivity.

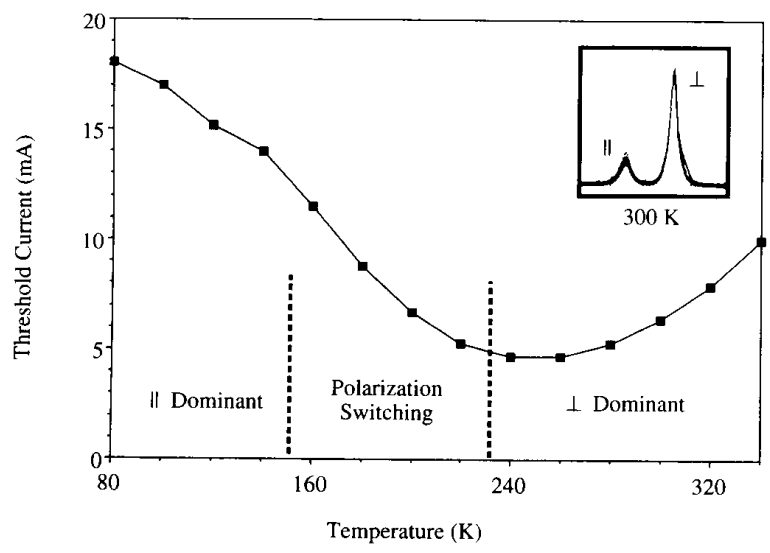

Fig. 3. Injection current and dominant polarization at threshold versus substrate temperature for a GaAs VCSEL. Note that the eigen polarizations, denoted as $\perp$ and $\|$, are not aligned along the $\langle 110\rangle$ crystal directions. The inset shows the spectral splitting $(9.9 \mathrm{GHz})$ between the eigen polarizations of the Gaussian mode.

and different wafers, we find the equilibrium spectral splitting between the polarization cavity resonances vary only slightly 


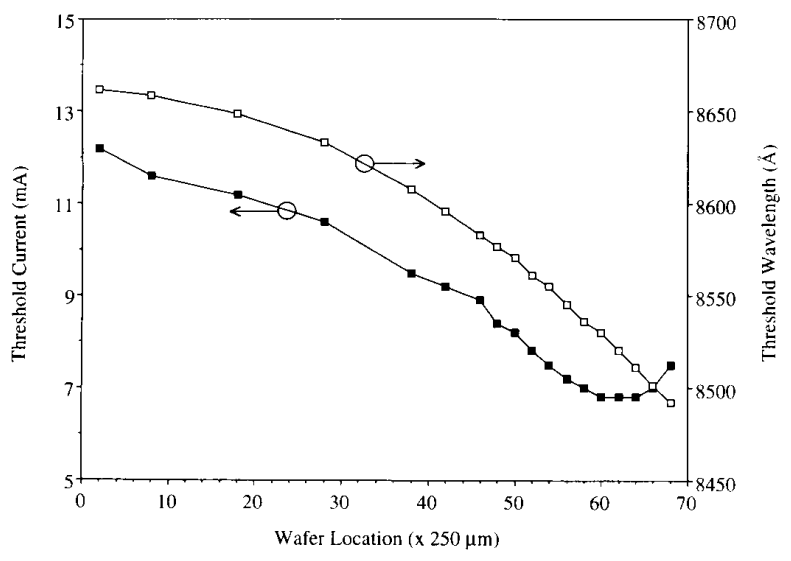

(a)

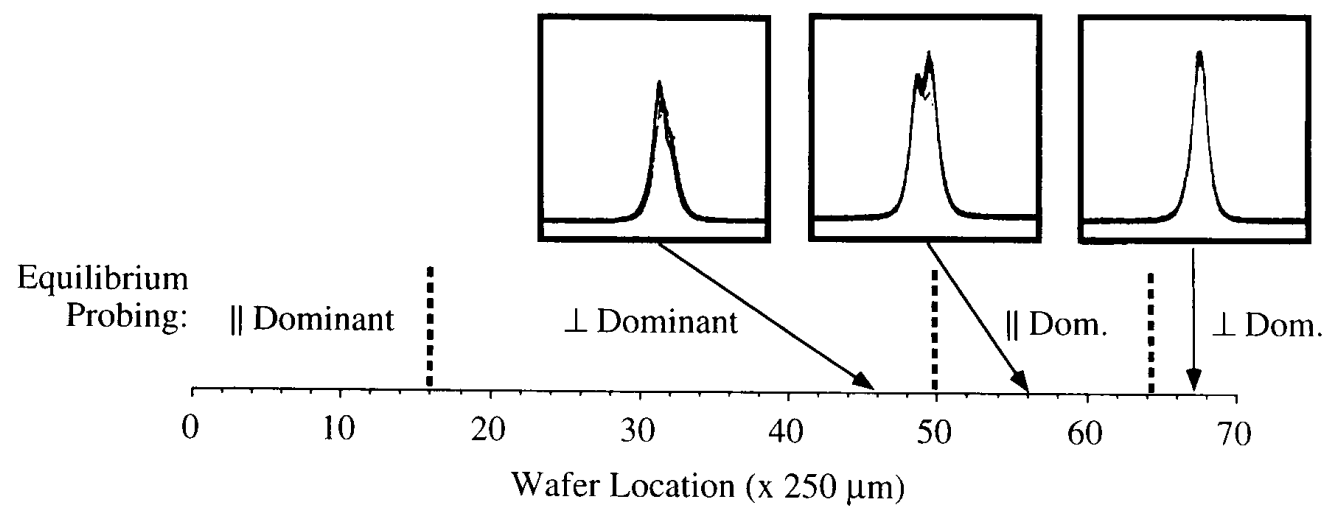

(b)

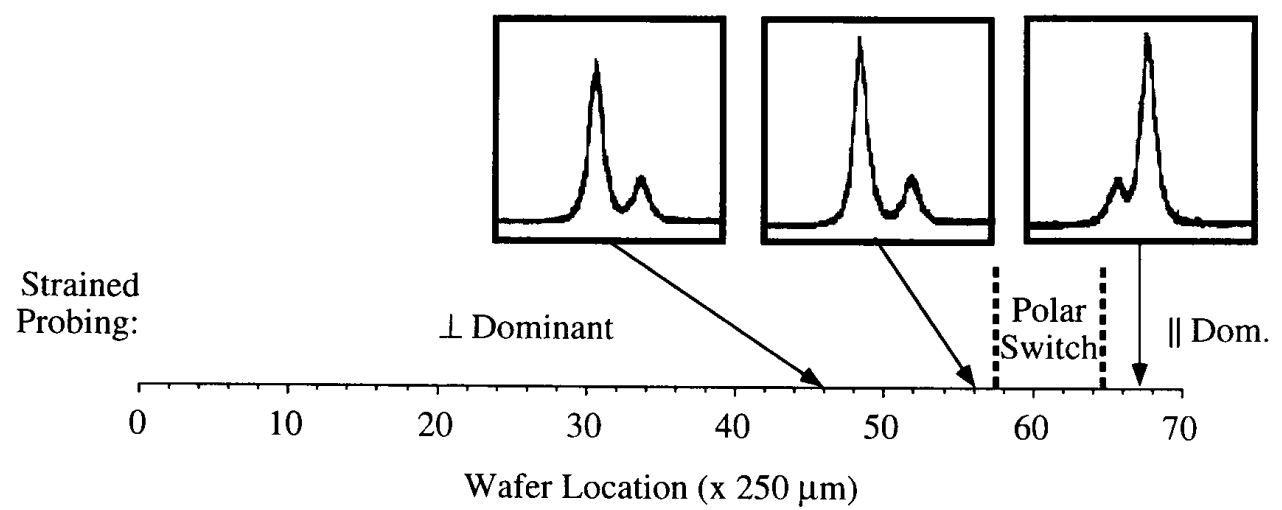

(c)

Fig. 4. (a) Injection current and wavelength at threshold versus wafer location for GaAs VCSEL's. The origin corresponds to the wafer center. (b) The dominant VCSEL polarization (denoted as $\perp$ and $\|)$ versus wafer location under equilibrium probing conditions. The insets show small or no spectral splitting $(<1.3$ $\mathrm{GHz}$ ) between the eigen polarization resonances of the threshold spatial mode. (c) The dominant VCSEL polarization versus wafer location with strain applied from probe tip pressure. The insets show the increased spectral splitting $(3.4-6 \mathrm{GHz})$ ) between the eigen polarization resonances of the threshold spatial mode.

between neighboring VCSEL's on a given sample, but can differ significantly between different samples fabricated at different times, even when from the same wafer.

\section{Discussion}

The gain-dependence of the polarization power partitioning is observed in both the InGaAs and GaAs VCSEL's and thus cannot be related to structural differences. Instead, the polarization power partitioning and the $I_{\mathrm{th}}$ dependence on wafer location or substrate temperature are related to the relative spectral alignment of the cavity resonances with the laser gain spectrum. In Figs. 2-4, the minimum $I_{\mathrm{th}}$ occurs at the wafer location or substrate temperature where the cavity resonance and the peak laser gain are optimally aligned. When 


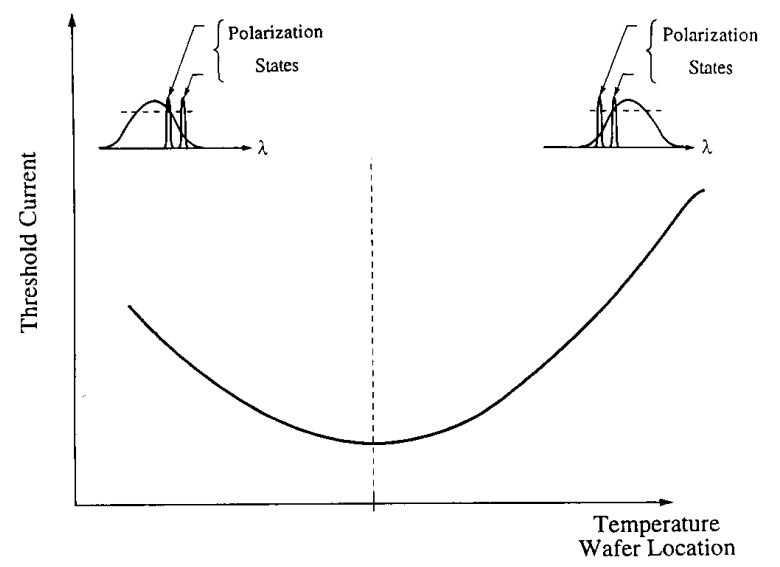

Fig. 5. Schematic of threshold current versus substrate temperature or device location on a nonuniform VCSEL wafer (not to scale). The insets depict the relative spectral alignment of the laser gain with the nondegenerate eigen polarization cavity resonances of the threshold mode (splitting greatly exaggerated) which produces the polarization gain-dependence.

the gain is blue- or red-shifted relative to the cavity resonance, greater injection is required to achieve lasing [16]. Due to the spectral splitting of the fundamental mode eigen polarizations, under the condition of blue-shifted gain, the shorter wavelength eigen polarization will experience enhanced gain overlap, while for red-shifted gain, the longer wavelength polarization state will dominate. This is schematically depicted in Fig. 5, where the insets show the differing gain alignment with the nondegenerate polarization cavity resonances.

Moreover, at the condition of cavity resonance/peak gain alignment, the dominant polarization abruptly switches as the red-shifting gain sweeps through the polarization resonances due to ohmic heating with increasing current injection. Such polarization switching is apparent for the Gaussian mode and higher-order transverse modes in Fig. 1(b), and has also been reported elsewhere [2], [11]. Note the regions of polarization switching shown in Figs. 2-4 correspond to conditions where the shorter wavelength polarization resonance is dominant at threshold (i.e. slightly blue-shifted laser gain), and the gain moves through the longer wavelength polarization resonance before the onset of a higher-order transverse mode. Careful examination of the polarization switching with increasing injection current shows that the polarized output fluctuates significantly as power oscillates between the two states during the switching transition, although the total output remains constant [2]. Thus for polarization uniformity of VCSEL arrays, it is crucial that the polarization cavity resonances do not span the gain peak maximum in order to avoid polarization switching and polarization partitioning noise.

The polarization of the higher-order transverse modes are also influenced by the relative cavity resonance/gain overlap as well as by spectral and/or spatial hole burning. In Fig. 1 the first kinks in the total output power curves (denoted with arrows) indicate the onset of a higher-order spatial mode. Notice when the gain is red-shifted relative to the cavity resonances as in Fig. 1(a), the higher-order mode arises orthogonally polarized to the fundamental, presumably to better utilize the gain [3]. However, with a sufficient blueshift in the gain, the higher-order mode consistently arises with the same polarization as the fundamental as seen in Fig. 1(c). This indicates that spatial/spectral hole burning is of greater consequence under the condition of red-shifted gain, and/or the asymmetry of the gain spectrum affects the polarization partitioning of the higher-order mode. We find the number of transverse modes possessing the same polarization as the fundamental increases as the blue-shifted gain offset is increased above $20 \mathrm{~nm}$ for the InGaAs VCSEL's. Therefore, arrays with each element having its gain maximum blueshifted $\geq 20 \mathrm{~nm}$ from the cavity resonance will provide a single dominant polarization through the onset of multi-mode operation.

Comparing Fig. 4(b) and (c), we also conclude that the gain-dependence of the VCSEL polarization requires sufficient spectral splitting between the cavity resonance of each eigen polarization to produce enough gain difference to allow one or the other eigen polarization to dominate. Empirically for the GaAs VCSEL's, we observe a polarization gaindependence when the eigen polarizations are separated by $>3 \mathrm{GHz}(>0.07 \AA)$. If the splitting is less than this, often the polarization states have equal or nearly equal intensity. A determination of the gain difference between the nondegenerate eigen states is beyond the scope of this paper, but is presumably small and dependent on the active medium. Although the splitting between the eigen polarizations does not seem to have an electrooptic origin, we have determined that locally applied stress, such as from a probe tip placed near the laser aperture (within $\approx 100 \mu \mathrm{m}$ ), will influence the splitting. Hence, residual stress in the VCSEL epilayers resulting from growth or device fabrication may produce the polarization birefringence found in a given sample. Local stress is also found to effect the polarization power partitioning, when the spectral splitting between the eigen polarizations is small ( $\leq 1 \mathrm{GHz}$ for GaAs VCSEL's). This is consistent with previous findings that anisotropic stress near the active region will influence the laser gain [5]. In any event, adequate spectral splitting between the eigen polarizations appears to produce sufficient gain differences to account for the observed gaindependence of the VCSEL polarization.

\section{CONCLUSION}

In summary, we have described the gain-dependence of the polarization partitioning in gain-guided infra-red VCSEL's. The power partitioning between the eigen polarizations is shown to be correlated with the spectral alignment of each polarization cavity resonance with the laser gain spectrum. This polarization gain-dependence requires the eigen polarizations to be spectrally nondegenerate to provide sufficient gain differences between the states. The laser gain differences arising from the spectral splitting of the eigen polarizations and the means to engineer this splitting are under investigation. Nevertheless, this gain-dependence provides a simple means for polarization control of VCSEL arrays. Specifically, by blue-shifting the gain $\geq 20 \mathrm{~nm}$ from the cavity resonances, the dominant eigen polarization for the fundamental and higher- 
order mode is fixed for all devices in an array of InGaAs VCSEL's.

In addition, at the condition of cavity resonance/gain spectral alignment, abrupt polarization switching and polarized output fluctuations are found. Although the induced polarization switching transitions in the VCSEL can be exploited for polarization modulation applications, it should noted that this phenomenon has a thermal origin (thermal shift of the band gap) and thus the modulation frequencies are found to be $<100 \mathrm{kHz}[10]$. From the opposite point of view, for continuous wave applications of the VCSEL, operating near the condition of polarization switching may introduce an unspecified output polarization state and concomitant polarization noise. Even though blue-shifted gain off-set has been shown to give higher maximum output power and greater temperature insensitivity for VCSEL's [17], it will also create the precise circumstance for polarization switching. Therefore, careful control of the laser cavity resonance/gain alignment will be necessary to achieve both high-performance and polarization control for VCSEL arrays.

\section{ACKNOWLEDGMENT}

The authors thank D. Richie and M. Hagerott-Crawford for discussions, J. Zolper and J. Escobedo for ion implantation, and S. Samora and K. M. Geib for technical assistance.

\section{REFERENCES}

[1] F S. Choa, Y. H. Lee, T. L. Koch, C. A. Burrus, B. Tell, J. L. Jewell, and R. E. Leibenguth, "High speed modulation of vertical-cavity surface emitting lasers," IEEE Photon. Technol. Lett., vol. 3, p. 697, 1991

[2] K. D. Choquette, D. A. Richie, and R. Liebenguth, "Temperature dependence of gain-guided vertical-cavity surface emitting lasers," Appl. Phys. Lett., vol. 64, p. 2062, 1994.

[3] C. J. Chang-Hasnain, J. P. Harbison, L. T. Florez, and N. G. Stoffel, "Polarization characteristics of quantum well vertical cavity surface emitting lasers," Electron. Lett., vol. 27, p. 163, 1991.

[4] D. Vakhshoori, "Symmetry considerations in vertical-cavity surface emitting lasers: Prediction of removal of polarization isotropicity on (001) substrates," Appl. Phys. Lett., vol. 65, p. 259, 1994.

[5] T. Mukaihara, F. Koyama, and K. Iga, "Engineered polarization control of GaAs/AlGaAs surface-emitting lasers by anisotropic stress from elliptical etched substrate hole," IEEE Photon. Technol. Lett., vol. 5, p. 133, 1993.

[6] A. Chavez-Pirson, H. Ando, H. Saito, and H. Kanbe, "Polarization properties of a vertical cavity surface emitting laser using a fractional layer superlattice gain medium," Appl. Phys. Lett., vol. 62, p. 3082, 1993.

[7] M. Shimuzi, T. Mukaihara, F. Koyama, and K. Iga, "Polarization control for surface emitting lasers," Electron. Lett., vol. 27, p. 1067, 1991.

[8] T. Mukaihara, N. Ohnoki, T. Baba, F. Koyama, and K. Iga, "A novel birefringent distributed Bragg reflector using a metal/dielectric polarizer for polarization control of surface emitting lasers," Jpn. J. Appl. Phys., vol. 33, p. L227, 1994.

[9] K. D. Choquette and R. E. Leibenguth, "Control of vertical-cavity laser polarization with anisotropic transverse cavity geometries," IEEE Photon. Technol. Lett., vol. 6, p. 40, 1994.

[10] K. D. Choquette, K. L. Lear, R. E. Leibenguth, and M. T. Asom, "Polarization modulation of cruciform vertical-cavity laser diodes," Appl. Phys. Lett., vol. 64, p. 2767, 1994.
[11] S. Jiang, Z. Pan, M. Dagenais, R. A. Morgan, and K. Kojima, "Highfrequency polarization self-modulation in vertical-cavity surface emit ting lasers," Appl. Phys. Lett., vol. 63, p. 3545, 1993.

[12] N. Badr, I. H. White, M. R. T. Tan, Y. M. Houng, and S. Y. Wang, "Enhanced polarization self-switching in a vertical-cavity surface emitting laser by gain saturation of transverse modes," Electron. Lett., vol. 30 , p. 1227,1994

[13] K. L. Lear, R. P. Schneider, Jr., K. D. Choquette, S. P. Kilcoyne, J. J. Figiel, and J. Zolper, "Vertical-cavity surface emitting lasers with $21 \%$ efficiency by metalorganic vapor phase epitaxy," IEEE Photon. Technol. Lett., vol. 6, p. 1053, 1994.

[14] M. Kondo, C. Anayama, T. Tanahashi, and S. Yamazaki, "Crystal orientation dependence of impurity dopant incorporation in MOVPEgrown III-V materials," J. Crys. Growth, vol. 124, p. 449, 1992.

[15] K. D. Choquette and R. E. Leibenguth, unpublished.

[16] B. Tell, K. F. Brown-Goebler, R. E. Leibenguth, F. M. Baez, and Y. H. Lee, "Temperature dependence of GaAs-AlGaAs vertical-cavity surface emitting lasers," Appl. Phys. Lett., vol. 60, p. 683, 1992.

[17] D. B. Young, J. W. Scott, F. H. Peters, M. G. Peters, M. L. Majewski, B. J. Thibeault, S. W. Corzine, and L. A. Coldren, "Enhanced performance of offset-gain high-barrier vertical-cavity surface emitting lasers," IEEE J. Quantum. Electron., vol. 29, p. 2013, 1993.

Kent D. Choquette, for a photograph and biography, see this issue, p. 615 .

Richard P. Schneider, Jr. received the B.S. degree in materials science and engineering from Washington State University in 1984, and the Ph.D. degree in the same field from Northwestern University in 1989.

His doctoral thesis research centered on the epitaxial growth of InAsP-InPbased strained-layer heterostructures, of interest for long-wavelength $(1.3-1.55 \mu \mathrm{m})$ optoelectronic devices, and the optical chracterization and modeling of their properties. Since 1989 he has been a Senior Member of the Technical Staff in the Center for Compound Semiconductor Technologies (CCST) at Sandia National Laboratories, Albuquerque, NM. His principal interests include the epitaxial design, growth and characterization of semiconductor heterostructures for optoelectronic devices. He has been closely tied to the effort to develop and refine the first AlGaInP-based visible light vertical-cavity surface-emitting laser diodes, and has also been a key member of Sandia efforts to further refine conventional IR VCSEL's.

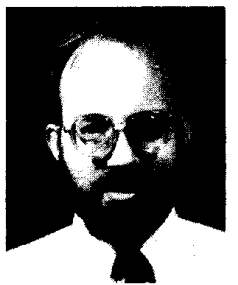

Kevin L. Lear ( $\left.{ }^{\prime} 83-M^{\prime} 88-S^{\prime} 88-M^{\prime} 90\right)$ received the B.S.E.E. degree from the University of Colorado in 1984 and M.S. and Ph.D. degrees in electrical engineering from Stanford University in 1985 and 1990 , respectively.

His thesis research was on resonant tunneling device structures and circuits. He is now researching optoelectronic devices at Sandia National Laboratory which he joined as a Senior Member of Technical Staff in 1990. His principal concentration is on enhancing the performance and exploring the physics of vertical-cavity surface emitting lasers including work on improving epitaxial mirrors, designing for high efficiency, and developing new structures. He also participates in the development of optoelectronic applications and quantum effect device research.

Ronald E. Leibenguth, photograph and biography not available at the time of publication. 